\title{
Klippel-Trenaunay-Weber syndrome in Palestinian neonate: case report
}

\begin{abstract}
Klippel-Trenaunay-Weber syndrome (KTWS) is a rare congenital disorder. The incidence of KTWS is 3-5/100000 live birth. It is characterized by port-wine stain, varicose veins, and bony and soft tissue hypertrophy affecting a limb. Most of the cases were diagnosed after the childhood when the clinical picture became clear and complete. Our baby was diagnosed just after birth; the right lower leg was hypertrophied with port-wine stained. Early diagnosis and multidisciplinary approach will improve the outcome and decrease the morbidity.
\end{abstract}

Keywords: Congenital condition " Haemangioma - Lymphatic dranage

Submitted Date: 07 May 2018; Accepted Date: 16 May 2018; Published Date: 18 May 2018

\section{Introduction}

KTWS is a rare congenital condition has clinical trial [1-3]:

- Cutaneous capillary haemangioma is mostly port-wine stain which is available since birth in the majority of cases.

- Soft tissue and bone hypertrophy of one limb, the lower limb is affected more than upper limb

\section{- Varicose veins}

The condition has a variable presentation. Not all clinical trial is available at birth, the hypertrophy of the limb and varicose maybe will take few years for one or two of them to be apparent. Internal organs and more than one limb may be are affected. KTWS could associate with arterial malformation and lymphatic drainage. KTWS has low blood velocity flow in malformed vessels whereas Parkes Weber's syndrome has high blood velocity flow in arteriovenous malformations. Male and female are equally affected. KTWS is sporadic disorder although limited cases were known to be familial. The etiology of the disorder is still unknown. KTWS is a clinical diagnosis which is confirmed by the imaging; MRI, Doppler ultrasound, and angiography.

\section{Case Report}

The parents were nonconsanguineous, they had no family history of congenital diseases, the mother was G8P7, the baby was full term female and the delivery was by normal vaginal delivery. Birth weight was 3700 grams and Apgar score was 8,9 at 1, 5 minute respectively. After birth on routine physical examination, the baby was found to have: the right lower limb is hypertrophied and it is covered by port wine stain (Figure 1).The other parts of physical examination were normal. Brain, abdominal ultrasound, and echocardiography studies were normal. Blood culture result was no growth. X-ray of lower limbs showed; right-sided hypertrophied soft tissue (Figure 2). Duplex ultrasonography of both lower limbs showed: right-sided limb muscles are hypertrophied when compared to the left-sided limb. The right-sided limb had prominent intramuscular vasculature mainly
Allam Fayez Abuhamda* Consultant Neonatologist, Shifa Women'S Hospital, Shifa Nicu, Ministry Of Health, Gaza, Palestine, Qatar *Author for correspondence: E-mail: allam570@yahoo.com 


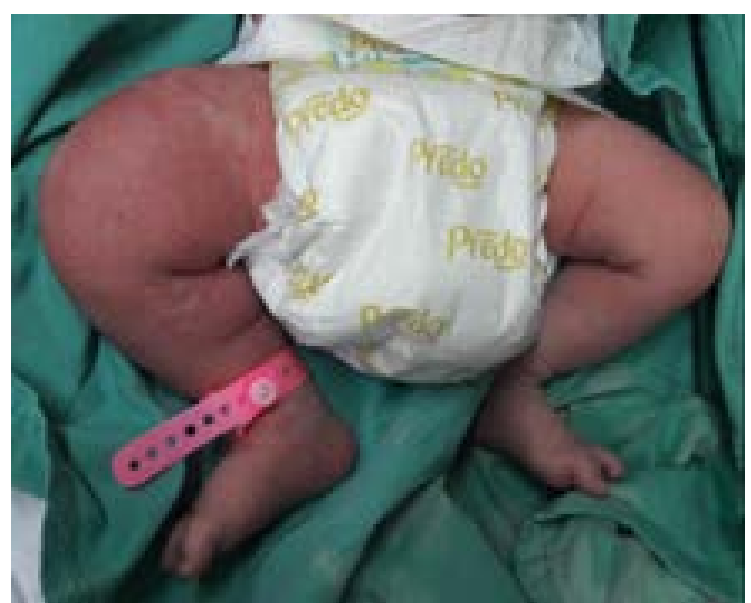

Figure 1: The right lower limb is hypertrophied and it is covered by port wine stain.

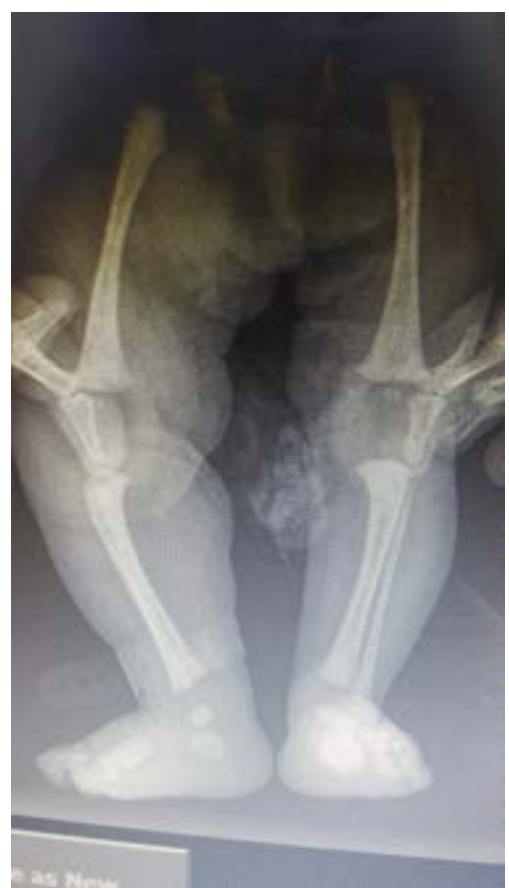

Figure 2: X-ray of lower limbs showed; right-sided hypertrophied soft tissue.

involving vastus medialis muscle. The multidisciplinary team consists of orthopaedic, vascular surgeon, plastic surgeon, social worker and neonatologist was involved in the management of the patient as KTWS case which needs close monitoring and follow up to prevent fatal complication and delay intervention.

\section{Discussion}

KTWS is a rare congenital disorder. The affected limb is characterized by clinical triad; cutaneous hemangioma, varicose veins and hypertrophied soft tissue and bone. The disorder has variable presentations. The condition was rarely diagnosed at the neonatal age as the clinical picture still incomplete [4]. The baby was full term female, on routine examination after birth, she found to have the right lower limb covered entirely port wine stain and hypertrophied compared to the left lower limb. The other parts of physical examination were normal. The X-ray showed the right lower limb was hypertrophied, the duplex ultrasonography of both lower limbs showed; the right lower limb is hypertrophied and had prominent intramuscular vasculature compared to the left lower limb. By Duplex ultrasonography as arteriovenous malformations (AVMs) were not available, Parkes Weber's syndrome was excluded. As the baby had not spinal or paraspinal (AVMs), seizure or scoliosis, CLOVE syndrome (Congenital lipomatous overgrowth, vascular malformations, and epidermal nevi) and Cobb syndrome were ruled out $[5,6]$. The clinical picture of the baby did not match the diagnosis of Sturge Weber syndrome as there was not facial port wine stain and brain ultrasound was normal [7]. The clinical picture of the baby is consistent with the KTWS diagnosis. There was two feature of the clinical triad; hypertrophied soft tissue and port wine stain of the right lower limb. From start social worker, plastic surgeon, pediatric orthopedic, vascular surgeon, and neonatologist were involved. Team plan recommended close monitoring and follow up to prevent morbid course like, cellulitis, limb length discrepancy, limb hypertrophy or atrophy and deep venous thrombosis.

\section{Conclusion}

A rare case of KTWS was diagnosed after birth and the case was managed by the multidisciplinary team which will make the prognosis is better with less complication. 


\section{References}

1. Bernatowicz R, Kuenzler R. An Unusual Case of KlippelTrenaunay-Weber Syndrome (P2. 267). Neurology 86: P2-267 (2016).

2. Kharat AT, Bhargava R, Bakshi V, Goyal A. Klippel-trenaunay syndrome: A case report with radiological review. Med J DY Patil Univ 9: 522 (2016).

3. Volz KR, Kanner CD, Evans J, Evans KD. Klippel-Trénaunay Syndrome. J Ultrasound Med 35.9 (2016): 2057-2065.
4. Kumar KS, Nanjappa MK. Klippel Trenaunay Syndrome: a rare case report in a neonate. Int J Contemp Pediatrics 4: 294-296 (2016).

5. Kasinathan A, Sankhyan N, Ahuja CK, Singhi P. CLOVE Syndrome. Indian J Pediatr 85: 79-80 (2017).

6. Pal P, Ray S, Chakraborty S, Dey S, Talukdar A. Cobb syndrome: A rare cause of paraplegia. Ann Neurosci 22: 191-193 (2015).

7. Desai S, Glasier C. Sturge-weber Syndrome. N Engl J Med 377: e11 (2017). 